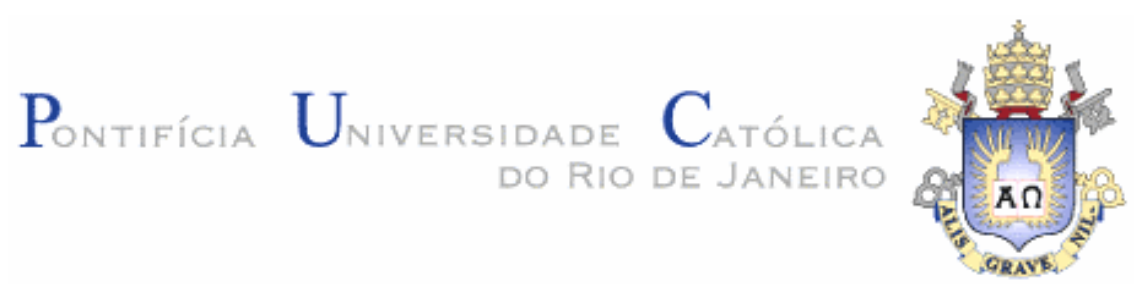

Ricardo Enrique Silva Cuentas

\title{
Análise da Estabilidade Dinâmica de Taludes de Solo
}

Dissertação apresentada como requisito parcial para obtenção do título de Mestre pelo Programa de PósGraduação em Engenharia Civil da PUC-Rio. Área de concentração: Geotecnia

Orientador: Celso Romanel 


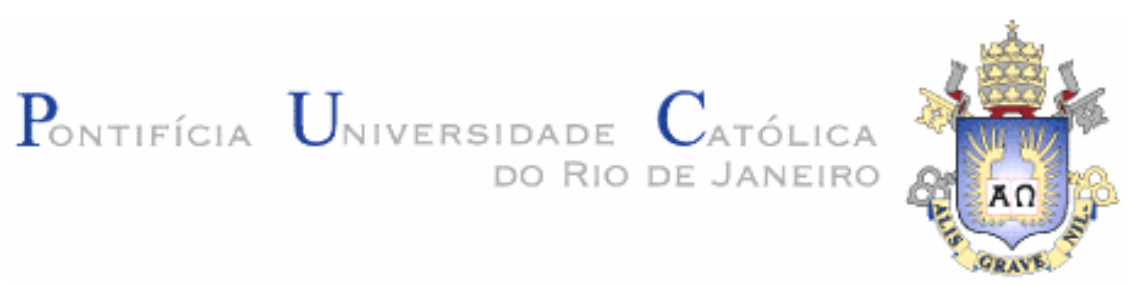

Ricardo Enrique Silva Cuentas

\section{Análise da Estabilidade Dinâmica de Taludes de Solo}

Dissertação apresentada como requisito parcial para obtenção do título de Mestre pelo Programa de PósGraduação em Engenharia Civil da PUC-Rio. Aprovada pela Comissão Examinadora abaixo assinada.

Celso Romanel Orientador PUC - Rio

Deane de Mesquita Roehl PUC - Rio

Araken Dumont Ramos Lima PUC - Rio

Ney Augusto Dumont Coordenador Setorial do Centro Técnico Científico da PUC-Rio

Rio de Janeiro, setembro de 2003 
Todos os direitos reservados. É proibida a reprodução total ou parcial do trabalho sem autorização da universidade, do autor e do orientador.

\section{Ricardo Enrique Silva Cuentas}

Graduou-se em Engenharia Civil pela Universidad Nacional de Ingenieria (UNI-Peru) em 1996. Desenvolveu durante a tese de graduação o programa denominado "Licua-Pro" para avaliação do potencial de liquefação e do dano produzido pelo fenômeno. Atuou na área geotécnica do Centro de Investigações Sísmicas e Mitigação de Desastres (CISMID-UNI). Ingressou em 2001 no curso de mestrado em Engenharia Civil da Pontifícia Universidade Católica do Rio de Janeiro, na área de Geotecnia, desenvolvendo dissertação de mestrado na linha de pesquisa Geomecânica Computacional sobre o comportamento dinâmico de taludes de solo.

Ficha Catalográfica

Cuentas, Ricardo Silva

Análise da estabilidade dinâmica de taludes de solo / Ricardo Silva Cuentas; orientador: Celso Romanel. - Rio de Janeiro: PUC, Departamento de Engenharia Civil, 2003.

xiii, 116f. : il. ; $30 \mathrm{~cm}$

Dissertação (mestrado) - Pontifícia Universidade Católica do Rio de Janeiro, Departamento de Engenharia Civil.

Inclui referências bibliográficas.

1. Engenharia civil - Teses. 2. Estabilidade de taludes. 3. Análise sísmica. 4. Comportamento dinâmico de solos. 5. Taludes Costa Verde (Peru). I. Romanel, Celso. II. Pontifícia Universidade Católica do Rio de Janeiro. Departamento de Engenharia Civil. III Título. 
Para meu melhor amigo, DEUS, pelas muitas bênçãos na minha vida; para meus pais, Luis Alberto e Asteria, pelo grande amor, confiança e exemplo; para meus 7 irmãos, pelos inesquecíveis momentos de felicidade; para Patrícia, pelo seu amor e apoio desinteressado. 


\section{Agradecimentos}

A Deus, por todas as graças recebidas.

À Pontifícia Universidade Católica (PUC-Rio) e à CAPES por terem me concedido a valiosa oportunidade de realizar este trabalho.

Ao professor Celso Romanel, por sua orientação, confiança e apoio, meus sinceros agradecimentos.

Aos meus irmãos Luis, Blas, José, Rosa, Juan Manuel, Carmen e Maria, pelo grande amor, em todos os momentos.

A todos os amigos e companheiros de estudos do curso de Mestrado em Engenharia Civil da PUC-Rio.

A Mariella Cañari pelos dados sobre os taludes da Costa Verde (Lima-Peru).

Aos funcionários da Secretaria do Departamento de Engenharia Civil, em especial à Ana Roxo, pela atenção, dedicação e paciência com os alunos de pós-graduação, especialmente com os estrangeiros. 


\section{Resumo}

Cuentas, Ricardo Silva; Romanel, Celso. Análise da Estabilidade Dinâmica de Taludes de Solo. Rio de Janeiro, 2003. 129 p. Dissertação de Mestrado - Departamento de Engenharia Civil, Pontifícia Universidade Católica do Rio de Janeiro.

Esta dissertação apresenta uma comparação dos métodos utilizados para análise da estabilidade dinâmica de taludes de solo através de métodos pseudoestáticos (equilíbrio limite) e dinâmicos (método dos elementos finitos).

Com ambos os métodos foram estudadas as características de estabilidade de 47 perfis de taludes da Costa Verde, faixa litorânea na cidade de Lima, Peru, abrigando importante rodovia ladeada por $8,25 \mathrm{~km}$ de taludes íngremes e de grande altura. Nesta região a ocorrência de sismos é freqüente, devido ao fenômeno da subducção da placa de Nazca sob a placa Continental SulAmericana. O sismo de projeto foi estabelecido em relação a estudos regionais de risco sísmico e o acelerograma utilizado corresponde ao registrado no terremoto de Lima de 03 de outubro de 1974, normalizado para uma aceleração máxima de projeto de $0,33 \mathrm{~g}$.

Os resultado obtidos pelos métodos pseudo-estáticos e pelo método dos elementos finitos apresentam diferenças em alguns dos perfis analisados, provavelmente porque os métodos pseudo-estáticos geralmente consideram as forças de inércia constantes na fatia e sem mudança de sentido durante o período da excitação sísmica.

\section{Palavras - chave}

Estabilidade de taludes; análise sísmica; comportamento dinâmico de solos; taludes Costa Verde (Peru). 
Cuentas, Ricardo Silva; Romanel, Celso (advisor). Dynamic Stability Analysis of Earth Slopes. Rio de Janeiro, 2003. 129 p., M.Sc. Thesis - Department of Civil Engineering, Pontifícia Universidade Católica do Rio de Janeiro.

This thesis presents a comparison between the methods generally used for dynamic stability analysis of earth slopes, based either on a pseudo-static approach (limit equilibrium method) or on a dynamic approach (finite element method).

Forty-seven profiles from the Costa Verde slopes located in Lima, Peru, were analyzed with both classes of methods. In this region seismic activity is quite common, caused by subduction of the Nazca plate into the South American Plate. The design seism was established from regional studies of seismic risks and the accelerogram used in this research corresponds to the seismic records from a major earthquake that hit Lima on October $3^{\text {rd }}$, 1974, herein normalized with respect to the maximum design acceleration of $0,33 \mathrm{~g}$.

The results obtained with pseudo-static and the finite element methods presented differences in some specific soil profiles, probably because the pseudostatic methods admit that the inertial forces are constants through a soil slice and there are no changes in direction of the dynamic forces during the period of seismic excitation.

\section{Keywords}

Slope stability; seismic analyses; dynamic soil behavior; Costa Verde (Peru) slopes. 


\section{Sumário}

1 INTRODUÇÃO

2 Análise pseudo-estática 18

3 Análise sísmica $\quad 47$

3.1. Método de Newmark (1965) 47

3.2. Método dos elementos finitos 50

3.2.1 Tipos de análise de estabilidade de taludes por elementos finitos 51

4 Fundamentos de análise sísmica 69

4.1. Tipos de sismos $\quad 69$

4.2. Parâmetros sismológicos $\quad 71$

4.3. Ondas planas de tensão (elásticas) 72

4.4 Equação do movimento $\quad 75$

4.5 Características do programa computacional Plaxis v.8 79

4.5.1 Integração no tempo 79

$\begin{array}{ll}\text { 4.5.2. Fator de segurança } & 80\end{array}$

5 Estabilidade sísmica dos taludes da Costa Verde 81

5.1. Localização 81

5.2. Propriedades dos solos 84

5.3. Estudo sísmico da área da Costa Verde 85

5.4. Sismo de projeto 86

5.5. Análise sísmica por métodos pseudo-estáticos 87

5.6. Análise sísmica pelo método dos elementos finitos 90

6 Conclusões e sugestões 100

$\begin{array}{ll}\text { Referências bibliográficas } & 101\end{array}$ 
Apêndice

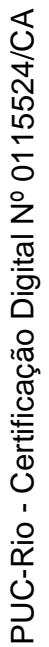




\section{Lista de Símbolos}

\begin{tabular}{|c|c|}
\hline$\alpha_{\mathrm{i}}$ & Ângulo de BXC com a horizontal \\
\hline$\beta$ & Ângulo do talude. \\
\hline$\beta_{\mathrm{REF}}$ & Inclinação dos reforços com a horizontal \\
\hline$\lambda$ & Fator de escala desconhecido \\
\hline$\alpha_{R}$ e $\beta_{R}$ & Coeficientes de Rayleigh \\
\hline$\gamma$ & Peso específico do material do talude \\
\hline$\psi$ & Ângulo de dilatãncia \\
\hline$\psi_{i}^{\prime}$ & Ângulo de atrito levando-se em conta a segurança \\
\hline$\phi ’$ & Resistência ao cisalhamento em termos de tensão efetiva \\
\hline$\phi_{d}$ & Ângulo de atrito desenvolvido \\
\hline$\sigma=\frac{N}{\ell}$ & Tensão média na base da fatia \\
\hline$\sigma^{\prime}$ & Tensão normal efetiva \\
\hline$\tau_{f}$ & Resistência ao cisalhamento \\
\hline$c, \phi$ & Parâmetros de resistência \\
\hline$c^{\prime}, \phi^{\prime}$ & Parâmetros de resistência em termos de tensões efetivas \\
\hline$c_{d}$ & Coesão desenvolvida \\
\hline $\bar{c}_{i}=\frac{c_{i}^{\prime}}{F S}$ & Coesão minorada pelo fator de segurança FS \\
\hline$v$ & Coeficiente de Poisson \\
\hline$a_{o}$ & Constante \\
\hline $\mathrm{a}$ & Aceleração \\
\hline$A 1, A 2$ & Forças hidrostáticas \\
\hline$b$, & Largura da fatia \\
\hline
\end{tabular}



$b_{i} \quad$ Largura de fatia
C Coesão
$C_{o} \quad$ Coesão no topo do talude
$\mathrm{C}_{\mathrm{u}} \quad$ Coesão não drenada ao longo da superfície de ruptura
C Matriz de amortecimento
D Fator da profundidade
D Força aplicada na superfície
$\overline{r u}_{i}, \bar{\phi}_{i}^{\prime}, c_{i}$
Valores médios dos parâmetros para a seção i
c' Coesão
$\mathrm{e}_{\mathrm{h}} \quad$ Excentricidade da carga $\mathrm{P}_{\mathrm{h}}$ em relação a $\mathrm{O}$
$E_{i}, E_{i+1} \quad$ Forças normais entre fatias, atuando nas seções i e i+1
E1, E2 Componente horizontal das forças entre as fatias
$E_{\text {ode }} \quad$ Módulo de elasticidade longitudinal confinado
$\mathrm{f}_{\text {arranc }} \quad$ Forca de arrancamento por metro (obtida em ensaios de campo)
F $\quad$ Vetor de carregamento
FS Fator de segurança
G Módulo de cisalhamento
$h_{i} \quad$ Altura de fatia
$\mathrm{H} \quad$ Altura do talude
K Matriz de rigidez
$K_{0} \quad$ Coeficiente en repouso
$\mathrm{K}_{\mathrm{h}} \quad$ Coeficiente sísmico ou aceleração horizontal média
$\mathrm{K}_{\mathrm{h}} \mathrm{W} \quad$ Força de inércia
$K_{v} \quad$ Aceleração vertical média
$\mathrm{l}_{\mathrm{i}} \quad$ Comprimento do arco BC 


\begin{tabular}{|c|c|c|}
\hline & $\ell$ & Comprimento da base da fatia \\
\hline & $1_{\text {REFj }}^{\prime}$ & Comprimento de reforço j além da superfície de ruptura \\
\hline & $m$ & Número de estabilidade \\
\hline & $M$ & Matriz de massa \\
\hline & $\mathrm{M}_{\mathrm{h}}$ & Momento da componente horizontal $\left(\mathrm{P}_{\mathrm{h}}\right)$ da carga externa \\
\hline & $N$ & Força normal à base da fatia \\
\hline & $\mathrm{N}_{1}, \mathrm{~N}_{2}$ & Números de estabilidade \\
\hline & $\mathrm{N}^{\prime}$ & Força normal efetiva \\
\hline & $\mathrm{P}_{\mathrm{V}}$ & Componente vertical da carga externa \\
\hline & $\mathrm{P}_{\mathrm{REFj}}$ & Força referente ao reforço $\mathrm{j}$ \\
\hline & Q & Função especial / Sobrecarga externa \\
\hline $\begin{array}{l}5 \\
0 \\
01\end{array}$ & $Q$ & Resultante das forças paralelas na fatia $\left(\mathrm{Z}_{\mathrm{i}}\right)$ \\
\hline$\frac{\frac{5}{\pi}}{\frac{\pi}{\sigma}}$ & $\mathrm{R}$ & Raio do arco circular \\
\hline $\begin{array}{l}0 \\
\substack{\infty \\
\mathbb{R}_{n}}\end{array}$ & $S=T \ell$ & Força tangencial à base da fatia \\
\hline 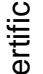 & $u$ & Vetor deslocamento \\
\hline (1) & $\stackrel{\circ}{u}$ & Velocidade \\
\hline نُ & $\ddot{u}$ & Aceleração \\
\hline & $\mathrm{u}$ & Poropressão \\
\hline & $\mathrm{U}=\mathrm{u} \cdot \ell$ & Força decorrente da poropressão \\
\hline & $\mathrm{Xi}$ & Forças verticais entre fatias \\
\hline & $\mathrm{x}_{\mathrm{i}}$ & Distância horizontal do centro da fatia a ponto $\mathrm{O}$ \\
\hline & $\mathrm{X}_{\mathrm{i}}, \mathrm{X}_{\mathrm{i}+1}$ & forças tangenciais entre fatias \\
\hline & $V_{p}$ & Velocidade de onda primária \\
\hline & $V_{s}$ & Velocidade de onda secundária \\
\hline & $\mathrm{S}_{\mathrm{m}}$ & Parcela mobilizada da resistência ao cisalhamento \\
\hline & $\mathrm{S}_{\mathrm{a}}$ & Resistência ao cisalhamento \\
\hline
\end{tabular}




$\begin{array}{ll}\mathrm{S}_{\mathrm{h}} & \text { Espaçamento horizontal entre reforços } \\ \mathrm{T}_{\mathrm{i}} & \text { Força cisalhante atuando na base da fatia } \\ \mathrm{U}_{\alpha} & \text { Força causada pela pressão da água nos poros } \\ \mathrm{U}_{\beta} & \text { Força causada pela pressão da água na superfície } \\ \mathrm{W} & \text { Peso da massa do solo } \\ \mathrm{W}_{\mathrm{i}} & \text { Peso da fatia de solo i } \\ Z & \text { Profundidade }\end{array}$

